\title{
Segment LLL-Reduction with Floating Point Orthogonalization.
}

\author{
Henrik Koy ${ }^{1}$ and Claus Peter Schnorr ${ }^{2}$ \\ 1 Deutsche Bank AG, Frankfurt am Main, henrik.koy@db.com \\ 2 Fachbereiche Mathematik und Informatik, Universität Frankfurt, PSF 111932, \\ D-60054 Frankfurt am Main, Germany. schnorr@cs.uni-frankfurt.de
}

\begin{abstract}
We associate with an integer lattice basis a scaled basis that has orthogonal vectors of nearly equal length. The orthogonal vectors or the $Q R$-factorization of a scaled basis can be accurately computed up to dimension $2^{16}$ by Householder reflexions in floating point arithmetic ( $f$ pa) with 53 precision bits.

We develop a highly practical $f p a$-variant the new segment LLLreduction of KOY AND SCHNORR [KS01]. The LLL-steps are guided in this algorithm by the Gram-Schmidt coefficients of an associated scaled basis. The new reduction algorithm is much faster than previous codes for LLL-reduction and performs well beyond dimension 1000.
\end{abstract}

Keywords. LLL-reduction, Householder reflexion, floating point arithmetic, stability, scaled basis, segment LLL-reduction, local LLL-reduction.

\section{Introduction.}

Practical algorithms for LLL-reduction compute the orthogonal vectors of a basis in floating point arithmetic $(f p a)$. The corresponding $f p a$-errors must be small otherwise the size-reduction included in the orthogonalization gets unstable. Up to dimension 250 Gram-Schmidt orthogonalization of an LLL-reduced basis can be done in $f p a$ with some correction steps [SE91]. Householder orthogonalization has better stability, as was shown in [RS96]. In practice it yields up to dimension 350 a sufficient $Q R$-factorization of the basis matrix - but this process gets unstable in dimension 400. [S88] presents a method for correcting the GramSchmidt coefficients using Schulz's method of matrix inversion. This method is provably stable but requires $O\left(n+\log _{2} M\right)$ precision bits, where $M$ bounds the Euclidean length of the basis vectors. It is useless for $f p a$ with 53 precision bits.

In this paper we associate with an integer lattice basis a scaled basis that has orthogonal vectors of nearly equal length. The orthogonal vectors or the $Q R$ factorization of a scaled basis can be accurately computed up to dimension $2^{16}$ by Householder reflexions in $f p a$ with 53 precision bits. We present an algorithm HRS that efficiently generates an associated scaled basis. We present a fpavariant of LLL-reduction where LLL-steps are guided by the orthogonal vectors of an associated scaled basis. In particular, size-reduction is done against the 
scaled basis. The weaker size-reduction does in practice not degrade the quality of the reduced basis.

We present a fpa-variant of segment LLL-reduction, a novel concept proposed in [KS01]. The algorithm segment sLLL performs scaled segment LLLreduction, so that all LLL-steps are guided by the orthogonaliation of an associated scaled basis. The algorithm segment sLLL is a very efficient reduction algorithm. Its efficiency comes from local LLL-reduction of two consecutive segments $B_{l-1}, B_{l}$ that is done by reducing the local matrix $R_{l}$ in $f p a$. To make this local LLL-reduction possible in the limits of $f p a$ it is necessary to bound the integer transformation matrix of the local LLL-reduction. For this we carefully prepare the local matrix $R_{l}$ of $B_{l-1}, B_{l}$ prior to local LLL-reduction.

In practice, segment sLLL is much faster than previous codes for LLLreduction. It performs well beyond dimension 1000 and provides lattice bases that are in practice of similar quality as LLL-reduced bases. For dimension 1000 and basis vectors with 400 bit integer coordinates, the new LLL-reduction takes only about 10 hours on a $800 \mathrm{MHz}$ PC. It is the first code for LLL-reduction that performs well beyond dimension 350 .

In this paper we focus on practical aspects related to $f p a$. A rigorous analysis of segment LLL-reduction in the model of integer arithmetic is in the companion paper [KS01]. For general background on fpa and Householder transformation see [LH95].

\section{Stability Properties of Householder Orthogonalization.}

For an introduction of LLL-reduced lattice bases and of our notation on lattices we refer to Section 2 of the companion paper [KS01]. For easy reference we recall Definition 1 and Theorem 1 from [KS01]. We let $\left.\delta \in] \frac{1}{4}, 1\right]$ and $\alpha=1 /\left(\delta-\frac{1}{4}\right)$.

Definition 1. An ordered basis $b_{1}, \ldots, b_{n} \in \mathbf{Z}^{d}$ of the lattice $L$ is LLL-reduced with $\left.\delta \in] \frac{1}{4}, 1\right]$ if it has properties $\mathbf{1 . 2 .}$.:

1. $\left|\mu_{j, i}\right| \leq 1 / 2 \quad$ for $1 \leq i<j \leq n$,

2. $\delta\left\|\widehat{b}_{i}\right\|^{2} \leq \mu_{i+1, i}^{2}\left\|\widehat{b}_{i}\right\|^{2}+\left\|\widehat{b}_{i+1}\right\|^{2} \quad$ for $i=1, \ldots, n-1$.

Theorem 1. A basis $b_{1}, \ldots, b_{n}$ of lattice $L$ that is LLL-reduced with $\delta$ satisfies:

1. $\left\|b_{i}\right\|^{2} \leq \alpha^{n-1} \lambda_{i}^{2}$ and $\left\|b_{1}\right\|^{2} \leq \alpha^{i-1}\left\|\widehat{b}_{i}\right\|^{2} \quad$ for $i=1, \ldots, n$,

2. $\left\|b_{1}\right\|^{2} \leq \alpha^{\frac{n-1}{2}}(\operatorname{det} L)^{\frac{2}{n}}$ and $\left\|\widehat{b}_{n}\right\|^{2} \geq \alpha^{-\frac{n-1}{2}}(\operatorname{det} L)^{\frac{2}{n}}$.

Accuracy of Householder reflexions. Consider the $Q R$-factorization $B=Q R$ of the basis matrix $B=\left[b_{1}, \ldots, b_{n}\right] \in \mathbf{Z}^{d \times n}$, where $Q \in \mathbf{R}^{d \times d}$ is an orthogonal matrix and $R=\left[r_{i, j}\right]=\left[\mathbf{r}_{1}, \ldots, \mathbf{r}_{n}\right] \in \mathbf{R}^{d \times n}$ is an upper triangular matrix, $r_{i, j}=0$ for $i>j$. We have $\mu_{j, i}=r_{i, j} / r_{i, i}$ and $\left|r_{i, i}\right|=\left\|\widehat{b}_{i}\right\|$. The vector $\mathbf{r}_{l}$ is the orthogonal transform of $b_{l}$. 
Consider the process of orthogonalization of a basis matrix $B=\left[b_{1}, \ldots, b_{n}\right]$. In ideal arithmetic we get the $Q R$-factorization $B=Q R$ by a sequence of Householder transformations

$$
C_{1}:=B, C_{j+1}:=Q_{j} C_{j} \text { for } j=1, \ldots, n,
$$

where $Q_{j} \in \mathbf{R}^{d \times d}$ is an orthogonal matrix - an Householder reflexion that produces zeros in positions $j+1$ through $d$ of column $j$ of $Q_{j} C_{j}$. Thus $C_{j+1} \in \mathbf{R}^{d \times n}$ is upper triangular in the first $j$ columns. Finally, $R=C_{n+1}, Q=$ $Q_{n} \cdots Q_{1}$.

In actual computation, however, we use floating point operations

$$
\bar{C}_{1}=f l(B), \bar{C}_{j+1}:=f l\left(\bar{Q}_{j} \bar{C}_{j}\right) \text { for } j=1, \ldots, n .
$$

We assume the standard fpa-model of WILKINSON, see [LH95, p. 85] for details. Let $0<\eta \ll 1$ be the relative precision - each floating point operation induces a normalized relative error bounded in magnitude by $\eta .{ }^{1}$ In this model, it has been shown [LH 95, p. 87, formula (15.38)] that

$$
\left\|\bar{C}_{j+1}-Q_{j} \cdots Q_{1} B\right\|_{F} \leq(6 d-3 j+40) j \eta\|B\|_{F}+O\left(\eta^{2}\right),
$$

where $\|A\|_{F}=\left(\sum_{i, j} a_{i, j}^{2}\right)^{\frac{1}{2}}$ denotes the Frobenius NORM of the matrix $A=$ $\left[a_{i, j}\right]$. In the following we neglect the low order term $O\left(\eta^{2}\right)$. Thus, in actual computation we get $\bar{R}=\left[\bar{r}_{i, j}\right]=\bar{C}_{n+1}$ satisfying for $n \geq 14$.

$$
\|\bar{R}-R\|_{F} \leq 6 d n \eta\|B\|_{F} .
$$

It follows that the approximate Gram-Schmidt coefficients $\bar{\mu}_{j, i}=\bar{r}_{i, j} / \bar{r}_{i, i}$ satisfy for $i<j$

$$
\left|\bar{\mu}_{j, i}-\mu_{j, i}\right| \leq 6 d j \eta\left\|b_{1}, \ldots, b_{j}\right\|_{F} /\left(\left|r_{i, i}\right|(1-\varepsilon)\right)
$$

provided that $\left|\bar{r}_{i, i}-r_{i, i}\right| \leq \varepsilon\left|r_{i, i}\right|$. Therefore, we get from inequality (2) the

Lemma 1. Inequality (3) holds provided that

$$
6 d j \eta\left\|b_{1}, \ldots, b_{j}\right\|_{F} \leq \varepsilon\left|r_{i, i}\right| .
$$

Instability of Householder QR-factorization for dimension 400. Inequalities (1), (2), (3) are rather sharp. To combine Householder transformation and sizereduction we need accurate coefficients $\mu_{j, i}$. Condition (4) characterizes the stability of Householder transformation - stability requires that $6 d n \eta\|B\|_{F}<$ $\min _{i}\left|r_{i, i}\right|$. On the other hand, Theorem 2 shows that random LLL-reduced bases on the average satisfy $\left\|b_{1}\right\| \approx 1.1^{n-1}\left\|\widehat{b}_{n}\right\|$. For dimension $n=400$ and $\eta=2^{-53}$ this yields $6 d n \eta\left\|b_{1}\right\|_{F} \approx 6 d n \eta 1.1^{n-1}\left\|\widehat{b}_{n}\right\| \approx 4 \cdot 10^{6}\left\|\widehat{b}_{n}\right\|$. Inequality (4) is grossly violated. Therefore, Householder transformation of LLL-reduced bases is necessarily unstable in dimension 400 for $f p a$ with 53 precision bits.

Theorem 2. Consider a random LLL-reduced basis with random coefficients $\mu_{i+1, i} \in_{R}\left[-\frac{1}{2}, \frac{1}{2}\right]$ for $i=1, \ldots, n-1$, and let the inequalities 2. of Definition 1 be tight. Then $\left\|b_{1}\right\| \approx 1.1^{n-1}\left\|\widehat{b}_{n}\right\|$ holds on the average.

\footnotetext{
$\overline{1}$ standard double length, wired $f p a$ has 53 precision bits, $\eta=2^{-53}$.
} 
Proof. While $\left(\delta-\mu_{i+1, i}^{2}\right)\left\|\widehat{b}_{i}\right\|^{2} \leq\left\|\widehat{b}_{i+1}\right\|^{2}$ holds for LLL-reduced bases, the converse $\left(\delta-\varepsilon^{2}\right)\left\|\widehat{b}_{i}\right\|^{2} \geq\left\|\widehat{b}_{i+1}\right\|^{2}$ holds provided that $\delta\left\|\widehat{b}_{i}\right\|^{2}=\left\|\widehat{b}_{i+1}\right\|^{2}+\mu_{i+1, i}^{2}\left\|\widehat{b}_{i}\right\|^{2}$ and $\left|\mu_{i+1, i}\right| \leq \varepsilon$. The inequality $\left|\mu_{i+1, i}\right| \leq \varepsilon$ holds with probability $2 \varepsilon$ for random $\mu_{i+1, i} \in_{R}\left[-\frac{1}{2}, \frac{1}{2}\right]$. As $\int_{-\frac{1}{2}}^{\frac{1}{2}} 2 \varepsilon^{2} d \varepsilon=\frac{1}{6}$ and $\sqrt{\delta-\frac{1}{6}} \approx 1.1^{-1}$ holds for $\delta \approx 1$ we see that $\left\|b_{1}\right\| \approx 1.1^{n-1}\left\|\widehat{b}_{n}\right\|$ holds on the average.

\section{The Scaled Basis Matrix.}

Scaling is a well known method for improving the stability of $f p a$. We associate with an integer lattice basis $b_{1}, \ldots, b_{n}$ a scaled basis $b_{1}^{s}, \ldots, b_{n}^{s}$ that has orthogonal vectors of nearly equal length.

Definition 2. Let $b_{1}, \ldots, b_{n}$ be a basis of an integer lattice $L$. We call $b_{1}^{s}, \ldots, b_{n}^{s} \in$ $L$ an associated scaled basis with scaling factors $2^{e_{1}}, \ldots, 2^{e_{n}}-e_{1}, \ldots, e_{n} \in \mathbf{N}-$ if $b_{1}^{s}, \ldots, b_{n}^{s}$ form a size-reduced basis of a sublattice of $L$ satisfying

$$
\left\|b_{1}^{s}\right\| \leq 2^{e_{i}} \mid \widehat{b}_{i}\|=\| \widehat{b}_{i}^{s}\|<2\| b_{1}^{s} \| \quad \text { for } i=1, \ldots, n .
$$

We show in Theorem 3 that a given scaled basis yields accurate Gram-Schmidt coefficients by Householder reflexions in fpa. In Section 4 we show how to produce an associated scaled basis efficiently in fpa. In Sections 5 and 7 we use an associated scaled basis to guide LLL-reduction and segment LLL-reduction.

We can easily transform a basis $b_{1}, \ldots, b_{n}$ into an associated scaled basis $b_{1}^{s}, \ldots, b_{n}^{s}$ using exact arithmetic — first scale then size-reduce:

1. scaling. $e_{1}:=\left\lfloor\log _{2}\left(\max _{j}\left\|\widehat{b}_{j}\right\| /\left\|b_{1}\right\|\right)\right\rfloor, b_{1}^{s}:=2^{e_{1}} b_{1}$,

$$
\text { for } i=2, \ldots, n \text { do } e_{i}:=\max \left(0,\left\lceil\log _{2}\left\|b_{1}^{s}\right\| /\left\|\widehat{b}_{i}\right\|\right\rceil\right), \quad b_{i}^{s}:=2^{e_{i}} b_{i}
$$

2. size-reduction. for $j=2, \ldots, n$ for $i=j-1, \ldots, 1$ do $b_{j}^{s}:=b_{j}^{s}-\left\lceil\frac{\left\langle b_{j}^{s}, \hat{b}_{i}^{s}\right\rangle}{\left\|b_{i}^{s}\right\|^{2}}\right\rfloor b_{i}^{s} .{ }^{2}$

New Notation. For the remaining of the paper we let the column vector $\mathbf{r}_{\nu}$ of the $R$-matrix be related to the scaled vector $b_{\nu}^{s}$ rather than to the original vector $b_{\nu}$.

The size of scaled Gram-Schmidt coefficients. The coefficients $\mu_{\nu, j}^{s}$ of an arbitrary, unscaled vector $b_{\nu}=\widehat{b}_{\nu}+\sum_{j=1}^{\nu-1} \mu_{\nu, j}^{s} \widehat{b}_{j}^{s}$ are uniformly bounded:

Corollary 1. $\left|\mu_{\nu, j}^{s}\right|=\frac{\left|\left\langle b_{\nu}, \widehat{b}_{j}^{s}\right\rangle\right|}{\left\|b_{j}^{s}\right\|^{2}} \leq \frac{\left\|b_{\nu}\right\|}{\left\|b_{j}^{s}\right\|} \stackrel{(5)}{\leq} 2 \cdot \frac{\left\|b_{\nu}\right\|}{\left\|b_{1}^{s}\right\|}$

In contrast, LLL-reduced bases $b_{1}, \ldots, b_{\nu-1}$ satisfy $b_{\nu}=\sum_{j=1}^{\nu} \mu_{\nu, j} \widehat{b}_{j}$ with $\left|\mu_{\nu, j}\right|^{2} \leq$ $\frac{\left\|b_{\nu}\right\|^{2}}{\left\|b_{1}\right\|^{2}} \alpha^{j-1}$. The coefficient $\mu_{\nu, \nu-1}$ that enters first into size-reduction of $b_{\nu}$ tends to be very large due to the factor $\alpha^{\nu-1}$. A small relative error of $\mu_{\nu, \nu-1}$ confuses the size-reduction of $b_{\nu}$.

\footnotetext{
${ }^{2}$ Let $\lceil r\rfloor=\left\lceil r-\frac{1}{2}\right\rceil$ be the nearest integer to the real number $r$.
} 
Corollary 2. The basis $b_{1}^{s}, \ldots, b_{n}^{s}$ satisfies $\left\|b_{j}^{s}\right\| \leq \sqrt{j+3}\left\|b_{1}^{s}\right\|$ for $j=1, \ldots, n$.

Proof.

$$
\begin{aligned}
\left\|b_{j}^{s}\right\|^{2} & =\left\|\widehat{b}_{j}^{s}\right\|^{2}+\sum_{i=1}^{j-1}\left(\mu_{j, i}^{s}\right)^{2}\left\|\widehat{b}_{i}^{s}\right\|^{2} \\
& \leq\left\|\widehat{b}_{j}^{s}\right\|^{2}+(j-1) / 4 \max _{i<j}\left(\left\|\widehat{b}_{i}^{s}\right\|\right)^{2} \\
& \stackrel{(5)}{\leq} 4\left\|b_{1}^{s}\right\|^{2}+(j-1)\left\|b_{1}^{s}\right\|^{2}=(j+3)\left\|\widehat{b}_{1}^{s}\right\|^{2} .
\end{aligned}
$$

Next we study for a given scaled basis the accuracy of the approximate coefficients $\bar{\mu}_{j, i}^{s}$, computed in $f p a$ by Householder reflexions.

Theorem 3. The approximate $\bar{\mu}_{j, i}^{s}$ of $b_{1}^{s}, \ldots, b_{n}^{s}$ satisfy $\left|\bar{\mu}_{j, i}^{s}-\mu_{j, i}^{s}\right| \leq \varepsilon /(1-\varepsilon)$ for $\varepsilon=6 d j^{2} \eta$.

Proof. By scaling and size-reduction we have for $j \neq 2:^{3}$

$$
\left\|b_{j}^{s}\right\|^{2} \leq \frac{1}{4} \sum_{i=1}^{j-1}\left\|\widehat{b}_{i}^{s}\right\|^{2}+\left\|\widehat{b}_{j}^{s}\right\|^{2} \leq \frac{j+3}{4} \max _{i \leq j}\left\|\widehat{b}_{i}^{s}\right\|^{2} \stackrel{(5)}{\leq} j \min _{i \leq j}\left\|\widehat{b}_{i}^{s}\right\|^{2} .
$$

Hence $\left\|b_{1}^{s}, \ldots, b_{j}^{s}\right\|_{F}=\left(\sum_{i=1}^{j}\left\|b_{i}^{s}\right\|^{2}\right)^{1 / 2} \leq \sqrt{j} \max _{i \leq j}\left\|b_{i}^{s}\right\| \leq j\left\|\widehat{b}_{i}^{s}\right\|$, and thus

$$
\left\|b_{1}^{s}, \ldots, b_{j}^{s}\right\|_{F} /\left\|\widehat{b}_{i}^{s}\right\| \leq j \quad \text { for } i=1, \ldots, j .
$$

Hence, Inequality (4) holds for $\varepsilon=6 d j^{2} \eta$. By Lemma 1 Inequality (3) holds for that $\varepsilon$ and thus $\left|\bar{\mu}_{j, i}^{s}-\mu_{j, i}^{s}\right| \stackrel{(3)}{\leq} 6 d j \eta\left\|b_{1}^{s}, \ldots, b_{j}^{s}\right\|_{F} /\left(\left|r_{i, i}\right|(1-\varepsilon)\right) \stackrel{(6)}{\leq} 6 d j^{2} \eta /(1-\varepsilon)=$ $\varepsilon /(1-\varepsilon)$.

Stability up to dimension $2^{16}$. Consider Theorem 3 in the case $j \leq n=d=2^{16}$ and $\eta=2^{-53}$. Then we have $\varepsilon \leq 6 n^{3} \eta \leq 0.19$ and $\varepsilon /(1-\varepsilon) \leq 0 . \overline{2} 4$. Therefore, Householder reflexions yield up to dimension $2^{16}$ sufficiently accurate GramSchmidt coefficients for the basis $b_{1}^{s}, \ldots, b_{n}^{s}$.

\section{Orthogonalization via Scaling and Size-Reduction.}

Suppose we are given $b_{1}^{s}, \ldots, b_{\nu-1}^{s}, b_{\nu}$ and we want to produce a scaled vector $b_{\nu}^{s}$. At that point the $\mu_{j, i}^{s}$ of $b_{1}^{s}, \ldots, b_{\nu-1}^{s}$ are given with high accuracy. We iteratively transform $b_{\nu}$ into $b_{\nu}^{s}$ using better and better approximations of the $\mu_{\nu, i}^{s}$. The procedure HRS (Householder, Reduction, Scaling) iterates the following steps

1. the first $\nu-1$ Householder transformations $b_{\nu} \mapsto \bar{Q}_{\nu-1} \cdots \bar{Q}_{1} b_{\nu}$

2. size-reduction of $b_{\nu}$ against $b_{1}^{s}, \ldots, b_{\nu-1}^{s}$,

3. scaling of $b_{\nu}$ to $b_{\nu}^{s}$.

Steps 1. 2. must be iterated as the size of $b_{\nu}$ is by Inequality (2) crucial for the accuracy of Householder transformation. As the scaling increases the size of $b_{\nu}$ we scale in stages, repeating $\mathbf{1}$. 2. after each stage of scaling. Let

\footnotetext{
${ }^{3}$ In the following we neglect the exception $j=2$.
} 
$Q_{j}=I_{d}-2 v_{j} v_{j}^{\top}\left\|v_{j}\right\|^{-2}$, where $I_{d} \in \mathbf{Z}^{d \times d}$ is the identity matrix and $v_{j} \in \mathbf{R}^{d}$ is the Householder vector associated with $Q_{j}$. Note that $x \mapsto Q_{j} x$ reflects $x$ at the hyperplane that is orthogonal to $v_{j}: Q_{j} v_{j}=-v_{j}, Q_{j} u=u$ for $u \perp v_{j}$. HRS is given for input the Householder vectors $v_{1}, \ldots, v_{\nu-1} \in \mathbf{R}^{d}$, the first $\nu-1$ columns $\overline{\mathbf{r}}_{1}, \ldots, \overline{\mathbf{r}}_{\nu-1}$ of the matrix $\bar{C}_{\nu}=\bar{Q}_{\nu-1} \cdots \bar{Q}_{1} \bar{C}_{1}$ and the computed scaling exponents $\bar{e}_{1}, \ldots, \bar{e}_{\nu-1}$. The operations on $b_{1}^{s}, \ldots, b_{\nu-1}^{s}$ are in exact integer arithmetic, the other operations are in $f p a$. Taking fpa-errors into account we relaxe size-reduction of $b_{\nu}^{s}$ to the relaxed condition $\left|\mu_{\nu, j}^{s}\right| \leq 0.52$.

Supressing backward rescaling. Upon entry of $\operatorname{HRS}(\nu)$, the scaled vectors $b_{1}^{s}, \ldots, b_{\nu-1}^{s}$ are given while $b_{\nu}^{s}, \ldots, b_{n}^{s}$ are unknown. At this stage the scaling factors $2^{\bar{e}_{1}}, \ldots, 2^{\bar{e}_{\nu-1}}$ correspond to the subbasis $b_{1}, \ldots, b_{\nu-1}$. If $\left\|\widehat{b}_{\nu}\right\|>\left\|\widehat{b}_{1}^{s}\right\|$ we would need to rescale $b_{i}^{s}$ by increasing $\bar{e}_{i}:=\bar{e}_{i}+\bar{e}$ and $b_{i}^{s}:=2^{\bar{e}} b_{i}^{s}$ for $\bar{e}:=\left\lfloor\log _{2}\left(\left\|\widehat{b}_{\nu}\right\| /\left\|\widehat{b}_{1}^{s}\right\|\right)\right\rfloor$ and $i=1, \ldots, \nu-1$. We suppress this backward rescaling. It is sufficient to store $\bar{e}$ and to do all subsequent size-reductions against $2^{\bar{e}} b_{i}^{s}$ rather than against $b_{i}^{s}$, i.e., we replace subsequent reduction steps $b:=b-\left\lceil\frac{\left\langle b, \widehat{b}_{i}^{s}\right\rangle}{\left\langle\widehat{b}_{i}^{s}, \widehat{b}_{i}^{s}\right\rangle}\right\rfloor b_{i}^{s}$ by the steps $b:=b-2^{\bar{e}}\left[\frac{2^{-\bar{c}}\left\langle b_{i}^{s}\right\rangle}{\left\langle\hat{b}_{i}^{s}, \widehat{b}_{i}^{s}\right\rangle}\right] b_{i}^{s}$. For simplicity, the program $\operatorname{HRS}(\nu)$ does not include the steps required in case that $\left\|\widehat{b}_{\nu}\right\|>\left\|\widehat{b}_{1}^{s}\right\|$.

$\operatorname{HRS}(\nu)$ (Householder transformation, Reduction and Scaling of $b_{\nu}$ )

INPUT $\quad b_{1}^{s}, \ldots, b_{\nu-1}^{s} \in \mathbf{Z}^{d}, \overline{\mathbf{r}}_{1}, \ldots, \overline{\mathbf{r}}_{\nu-1}, v_{1}, \ldots, v_{\nu-1} \in \mathbf{Q}^{d}, \bar{e}_{1}, \ldots, \bar{e}_{\nu-1} \in \mathbf{Z}$

OUTPUT $b_{\nu}^{s}$ (size-reduced against $b_{1}^{s}, \ldots, b_{\nu-1}^{s}$ ), $v_{\nu}, \overline{\mathbf{r}}_{\nu}, \bar{e}_{\nu}$

1. $\bar{e}_{\nu}:=0, b_{\nu}^{s}:=b_{\nu}$

2. $\overline{\mathbf{r}}_{\nu}:=\bar{Q}_{\nu-1} \cdots \bar{Q}_{1} \cdot b_{\nu}^{s}$

3. size-reduce $b_{\nu}^{s}$ against $b_{1}^{s}, \ldots, b_{\nu-1}^{s}$

$$
\begin{aligned}
\text { for } & i=\nu-1, \ldots, 1 \text { do } \\
& \bar{\mu}_{\nu, i}^{s}:=\bar{r}_{i, \nu} / \bar{r}_{i, i} \\
& \text { if }\left|\bar{\mu}_{\nu, i}^{s}\right| \geq 0.51 \text { then } b_{\nu}^{s}:=b_{\nu}^{s}-\left\lceil\bar{\mu}_{\nu, i}^{s}\right\rfloor b_{i}^{s}, \overline{\mathbf{r}}_{\nu}:=\overline{\mathbf{r}}_{\nu}-\left\lceil\bar{\mu}_{\nu, i}^{s}\right\rfloor \overline{\mathbf{r}}_{i}
\end{aligned}
$$

4. if $\exists i:\left|\bar{\mu}_{\nu, i}^{s}\right| \geq 2^{10}$ then go to 2 .

5. $\tau:=\left(\sum_{i=\nu}^{d} \bar{r}_{i, \nu}^{2}\right)^{\frac{1}{2}}, \widetilde{e}:=\min \left(\left\lceil\log _{2}\left(\left\|b_{1}^{s}\right\| / \tau\right)\right\rceil, 30\right)$

6. if $\tilde{e}>0$ then $\bar{e}_{\nu}:=\bar{e}_{\nu}+\widetilde{e}, b_{\nu}^{s}:=2^{\tilde{e}} b_{\nu}^{s}$ go to 2 .

7. $\overline{\mathbf{r}}_{\nu}:=\bar{Q}_{\nu-1} \cdots \bar{Q}_{1} b_{\nu}^{s}, \quad \sigma:=\operatorname{sig}\left(\bar{r}_{\nu, \nu}\right), \quad \tau:=\left(\sum_{i=\nu}^{d} \bar{r}_{i, \nu}^{2}\right)^{\frac{1}{2}}$

$$
\begin{aligned}
& v_{\nu}:=\left(0, \ldots, 0, \bar{r}_{\nu, \nu}+\sigma \tau, \bar{r}_{\nu+1, \nu}, \ldots, \bar{r}_{d, \nu}\right)^{\top} \\
& \overline{\mathbf{r}}_{\nu}:=\left(\bar{r}_{1, \nu}, \ldots, \bar{r}_{\nu-1, \nu},-\sigma \tau, 0, \ldots, 0\right)^{\top} \quad \text { end }
\end{aligned}
$$

How HRS works. According to (5) the input vectors $b_{1}^{s}, \ldots, b_{\nu-1}^{s}$ satisfy $\left|r_{1,1}\right| \leq$ $\left|r_{i, i}\right|<2\left|r_{1,1}\right|$ for $i=1, \ldots, \nu-1$. Let $6 d \nu^{2} \eta<\frac{1}{2}$ so that Theorem 3 holds for $\varepsilon=\frac{1}{2}$. Then, by (2) and (6) we have that $\left|\bar{r}_{i, i}-r_{i, i}\right| \leq 6 d j^{2} \eta\left|r_{i, i}\right|$ for $i=1, \ldots, j$.

Reducing long $\left\|b_{\nu}\right\|$. By (2) the relative error of $\bar{r}_{i, \nu} /\left\|b_{\nu}\right\|$ in step 2 . is at most $6 d \nu \eta$. The subsequent size-reduction reduces the large $\bar{r}_{i, \nu}-$ in the equation 
$\left\|b_{\nu}\right\|^{2}=\sum_{i=1}^{d} r_{i, \nu}^{2}$ - to less than $\left|\bar{r}_{i, i}\right|$ in absolute value. Steps 2. 3. 4. decrease the $\left(\sum_{i=1}^{\nu-1} \bar{r}_{i, \nu}^{2}\right)^{\frac{1}{2}}$-part until the inequality $\left(\sum_{i=1}^{\nu-1} \bar{r}_{i, \nu}^{2}\right)^{\frac{1}{2}} \leq\left\|b_{1}^{s}, \ldots, b_{\nu-1}^{s}\right\|_{F}$ holds.

Upon entry of step 3 . the coefficients $\mu_{\nu, i}^{s}$ are uniformly bounded as $\left|\mu_{\nu, j}^{s}\right| \leq$ $2 \cdot \frac{\left\|b_{\nu}\right\|}{\left\|b_{1}^{s}\right\|}$, see Corollary 1. Size-reduction of Step 3. can temporarily increase the coefficients $\left|\mu_{\nu, j}^{s}\right|$ up to a factor $(3 / 2)^{\nu-j-1}$ in worst case. This could possibly make the size-reduction of step 3 . unstable in worst case. No such instability has been reported in practice, see Figure 1.

Scaling up small $\left\|\widehat{b}_{\nu}\right\|$. Let $\left\|b_{\nu}\right\| \leq\left\|b_{1}^{s}, \ldots, b_{\nu-1}^{s}\right\|_{F}$ and $\left|r_{\nu, \nu}\right| \leq \eta\left\|b_{\nu}\right\|$. By (2) and (6) we have after step 2. that $\left|\bar{r}_{\nu, \nu}-r_{\nu, \nu}\right|<6 d \nu \eta\left\|b_{1}^{s}, \ldots, b_{\nu-1}^{s}\right\|_{F} \leq$ $6 d \nu^{2} \eta\left\|b_{1}^{s}\right\|$. This yields a scaling factor $\tilde{e}$ for step 6 . so that $2^{\tilde{e}} \geq\left(6 d \nu^{2} \eta\right)^{-1}>\overline{1}$. Therefore, steps 2. to 6. increase $\left|\bar{r}_{\nu, \nu}\right|,|| b_{\nu}^{s}||$ until $\left|\bar{r}_{1,1}\right| \leq\left|\bar{r}_{\nu, \nu}\right| \approx\left\|b_{\nu}^{s}\right\|<2\left|\bar{r}_{1,1}\right|$.

Corollary 3. HRS $(\nu)$ produces a scaled vector $b_{\nu}^{s}$ satisfying $\left|\mu_{\nu, i}^{s}\right| \leq 0.52$ for $i=1, \ldots, \nu-1$ provided that size-reduction of Step 3 . remains stable.

Speeding up HRS. To speed up HRS replace the rounded values $\left\lceil\bar{\mu}_{\nu, i}^{s}\right\rfloor$ in step 3. by single precision integers consisting of the leading bits of $\left[\bar{\mu}_{\nu, i}^{s}\right\rfloor$. This way HRS becomes very fast.

The number of arithmetic steps. A matrix-vector multiplication $b_{\nu} \mapsto \bar{Q}_{1} b_{\nu}=$ $b_{\nu}-\frac{2 v_{1} v_{1}^{\top} b_{\nu}}{\left\|v_{1}\right\|^{2}}$ requires $2 d$ multiplications and one division - as usual we neglect the additions/subtractions. Thus, we get $\overline{\mathbf{r}}_{\nu}:=\bar{Q}_{\nu-1} \cdots \bar{Q}_{1} b_{\nu}$ using $2 \nu d$ multiplications/divisions. Size-reduction of step 3. requires $\nu d$ multiplications in exact arithmetic. In total, one round of steps 2. 3. 4. requires $2 \nu d$ multiplications/divisions in $f p a$ and $\nu d$ exact multiplications of long integers.

The number of rounds. In practice each round of steps 2. 3. 4. either decreases $\left(\sum_{i=1}^{\nu-1} r_{i, \nu}^{2}\right)^{\frac{1}{2}}$ by the factor $6 d \nu^{2} \eta$ or increases $\left\|\widehat{b}_{\nu}^{s}\right\|$ by the scaling factor $2^{\bar{e}_{\nu}} \geq$ $\left(6 d \nu^{2} \eta\right)^{-1}>1$ or does a combination of both, see the explanations for HRS. In practice $\mathbf{H R S}(\nu)$ requires $\log _{2}\left(\left\|b_{\nu}\right\| /\left\|\widehat{b}_{\nu}\right\|\right) /\left|\log _{2}\left(6 d \nu^{2} \eta\right)\right|$ rounds.

Practical Performance of HRS. Consider a random public-key basis of the GGH-cryptosystem [GGH97] of dimension 400. Figure 1 shows how HRS(400) decreases the coefficients $\left|\mu_{400, j}\right|$ of the last basis vector $b_{400}$. Using 53 bit $f p a$ each of five rounds decreases $\left|\mu_{400, j}\right|$ by a factor about $10^{9} \approx 2^{30}$. After each round all coefficients $\left|\mu_{400, j}\right|$ are of nearly equal size because the preceding orthogonal vectors have been scaled to nearly equal length $\left\|\widehat{b}_{j}^{s}\right\|$. After each of the first 4 rounds however, $\left|\mu_{\nu, j}^{s}\right|$ increases by about a factor 10 as $j$ decreases from 400 to 1 . This is due to the temporary increase of $\left|\mu_{\nu, j}^{s}\right|$ by a factor $(3 / 2)^{\nu-j-1}$ in worst case. Figure 1 shows that this temporary increase has little effect in practice.

Using 106 bit $f p a$ - instead of 53 bit $f p a$ - the five rounds of HRS(400) reduce to two rounds, and the running time of HRS reduces accordingly. Software implemented $f p a$ with 106 precision bits makes the reduction clearly faster than the standard $f p a$ with 53 precision bits. 


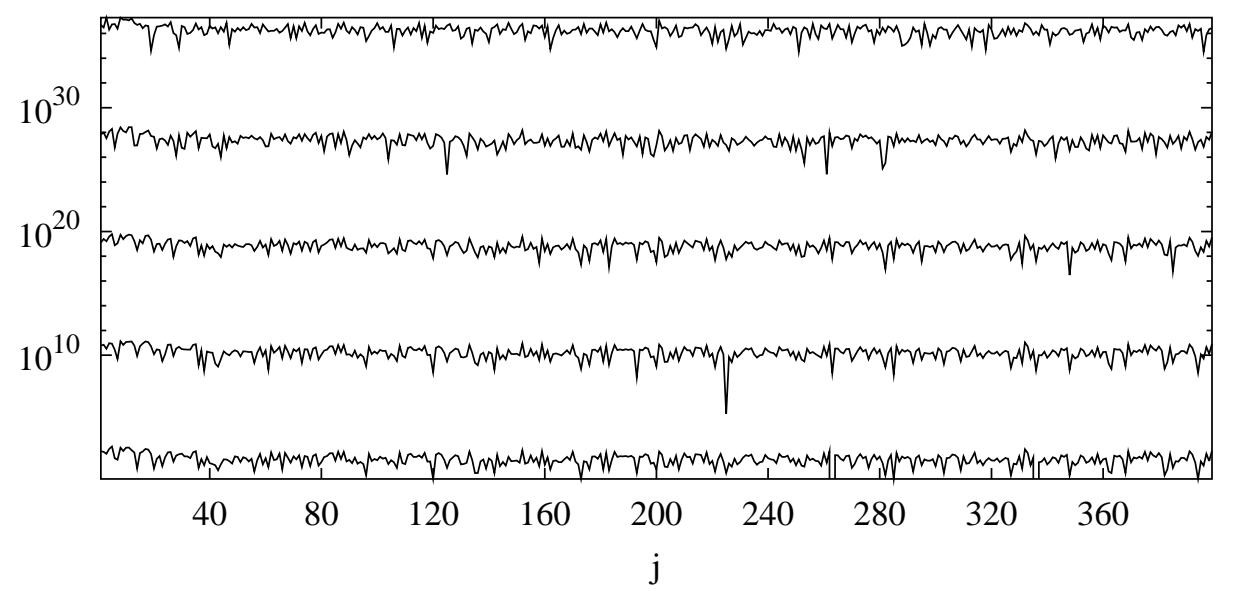

Fig. 1. Displayed are the values $\left|\mu_{400, j}\right|$ upon termination of each of five rounds. The $\left|\mu_{400, j}\right|$ are measured after step 2 of the next round - after new orthogonalization.

\section{Scaled LLL-Reduction.}

We introduce scaled LLL-reduced lattice bases, and we present an algorithm scaled LLL for scaled LLL-reduction. This algorithm is a useful prelude to the more complicated scaled segment LLL-reduction of Section 7.

Definition 3. We call a lattice basis $b_{1}, \ldots, b_{n} \in \mathbf{Z}^{d}$ scaled LLL-reduced if it has properties $\mathbf{1}$. 2 .:

1. There is an associated scaled basis $b_{1}^{s}, \ldots, b_{n}^{s}$ so that $b_{1}, \ldots, b_{n}$ is size-reduced against $b_{1}^{s}, \ldots, b_{n}^{s}$, i.e., $\quad\left|\frac{\left\langle b_{\nu}, \widehat{b}_{j}^{s}\right\rangle}{\left\|\widehat{b}_{j}^{s}\right\|^{2}}\right| \leq 0.52$ for $1 \leq i<j \leq n$

2. $\delta\left\|\widehat{b}_{i}\right\|^{2} \leq \alpha\left\|\widehat{b}_{i+1}\right\|^{2} \quad$ for $i=1, \ldots, n-1$.

We call a basis with property 1 . scaled-reduced. Importantly, the inequalities of Theorem 1 still hold for scaled LLL-reduced lattice bases. The weaker sizereduction does not affect these inequalities. Scaled LLL-reduced bases are in practice as good as LLL-reduced bases.

Next we present an algorithm scaled LLL that transforms a lattice basis into a scaled LLL-reduced basis of the same lattice. Scaled LLL guides the LLLsteps on the original basis by the orthogonalization of an associated scaled basis. So we keep and update two versions of the basis. LLL-transformations operate on the original basis. Size-reduction is done against the scaled basis. This form of LLL-reduction is quite stable as HRS yields an accurate $Q R$-factorization of the scaled basis.

The procedure HRS'. Local LLL-reduction of two basis vectors $b_{\nu-1}, b_{\nu}$ is guided by the orthogonal vector of a modified scaled vector $b_{\nu}^{s}$ - with a scaling factor 
$2^{\bar{e}_{\nu}}$ that coincides with the scaling factor $2^{\bar{e}_{\nu-1}}$ of $b_{\nu-1}$. We get the modified $b_{\nu}^{s}$ by the following variant HRS' of HRS: Set in Step 1. $\bar{e}_{\nu}:=\bar{e}_{\nu-1}$ and skip Step 6. Moreover, we let HRS' perform in Step 3. the same size-reduction steps $b_{\nu}^{s}:=b_{\nu}^{s}-\left\lceil\bar{\mu}_{\nu, \nu-1}^{s}\right\rfloor b_{\nu-1}^{s}, b_{\nu}:=b_{\nu}-\left\lceil\bar{\mu}_{\nu, \nu-1}^{s}\right\rfloor b_{\nu-1}$ on $b_{\nu}^{s}$ against $b_{\nu-1}^{s}$ and on $b_{\nu}$ against $b_{\nu-1}$. As a consequence we have

Lemma 2. The reduction coefficients of $b_{\nu}$ against $b_{\nu-1}$ and of $b_{\nu}^{s}$ against $b_{\nu-1}^{s}$ coincide in HRS' $(\nu)$, and thus $\mu_{\nu, \nu-1}=\mu_{\nu, \nu-1}^{s}$. The output vector $b_{\nu}$ of HRS' $^{\prime}(\nu)$ is size-reduced against $b_{\nu-1}$. The coefficients $r_{i, j}$ for $\nu-1 \leq i, j \leq$ $\nu$ associated with $b_{\nu-1}, b_{\nu}$ are proportional to the coefficients associated with $b_{\nu-1}^{s}, b_{\nu}^{s}$ with proportionality factor $2^{\bar{e}_{\nu}}$.

Scaled LLL (Algorithm for scaled LLL-reduction.)

INPUT $b_{1}, \ldots, b_{n} \in \mathbf{Z}^{d}, \delta$

OUTPUT $b_{1}, \ldots, b_{n}$ scaled LLL-reduced basis

1. $\nu:=1$

2. while $\nu \leq n$ do

3. $\quad$ if $\nu=1$ then $\operatorname{HRS}(1), \quad \nu:=2$

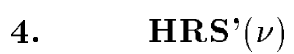

5. if $\delta \bar{r}_{\nu-1, \nu-1}^{2}>\bar{r}_{\nu-1, \nu}^{2}+\bar{r}_{\nu, \nu}^{2}$

then $\operatorname{swap} b_{\nu-1}, b_{\nu}, \quad \nu:=\nu-1$

else $\operatorname{HRS}(\nu), \nu:=\nu+1 \quad$ end

Scaling does not affect LLL-exchanges. By Lemma 2, the output vector $b_{\nu}$ of $\operatorname{HRS}^{\prime}(\nu)$ is size-reduced against $b_{\nu-1}$, i.e., $\left|\mu_{\nu, \nu-1}\right| \leq 0.52$. Moreover, the decision about swapping $b_{\nu-1}, b_{\nu}$ in Step $\mathbf{5}$. is the same as for the original LLLalgorithm - except for $f p a$-errors.

fpa-errors do not affect the LLL-exchanges. We see from $\left\|b_{\nu}^{s}\right\|^{2}=\sum_{i=1}^{d} r_{i, \nu}^{2}$ and (2) that the relative error of $\bar{r}_{\nu-1, \nu} /|| b_{\nu}^{s} \| \approx \bar{r}_{\nu-1, \nu} /\left|\bar{r}_{\nu, \nu}\right|$ and $\left|r_{\nu, \nu}\right| /|| b_{\nu}^{s} \| \mid$ is at most $6 d \nu \eta$. On the other hand, $\left|\bar{r}_{\nu-1, \nu-1}\right| \geq|| b_{1}^{s} \|$ holds by scaling. Therefore, the decision about swapping $b_{\nu-1}, b_{\nu}$ in Step $\mathbf{5}$. is correct for $6 d \nu \eta \ll 1$.

\section{Segment LLL-Reduction.}

We summarize the concept of segment LLL-reduced bases of the companion paper, for full coverage see [KS01].

Segments and local coordinates. Let the basis $b_{1}, \ldots, b_{n} \in \mathbf{Z}^{d}$ have dimension $n=k \cdot m$ and the $Q R$-factorization $\left[b_{1}, \ldots, b_{n}\right]=Q R$. We partition the basis-matrix $B$ into $m$ segments $B_{l}=\left[b_{k(l-1)+1}, \ldots, b_{k l}\right]$ for $l=1, \ldots, m$. Local reduction of two consecutive segments uses the coefficients of the submatrix $R_{l}:=\left[r_{k l+i, k l+j}\right]_{-k<i, j \leq k} \in \mathbf{R}^{2 k \times 2 k}$ of $R \in \mathbf{R}^{d \times n}$, corresponding to two consecutive segments $B_{l-1}, B_{l}$. We want to do most of the LLL-exchanges 
and the corresponding size-reduction in local coordinates of some $R_{l}$. Extra global transformations are required after local LLL-reduction. In order to minimize these global costs we introduce $k$-segment reduced bases. We let $D(l)=$ $\left\|\widehat{b}_{k(l-1)+1}\right\|^{2} \cdots\left\|\widehat{b}_{k l}\right\|^{2}$ denote the local Gramian determinant of segment $B_{l}$. We have that $D_{k l}=D(1) \cdots D(l)$.

Definition 4. We call a basis $b_{1}, \ldots, b_{n} \in \mathbf{Z}^{d}, n=k m, k$-segment LLL-reduced with $\left.\delta \in] \frac{1}{4}, 1\right]$ if it is size-reduced and satisfies for $\alpha=1 /\left(\delta-\frac{1}{4}\right)$ :

1. $\delta\left\|\widehat{b}_{i}\right\|^{2} \leq \mu_{i+1, i}^{2}\left\|\widehat{b}_{i}\right\|^{2}+\left\|\widehat{b}_{i+1}\right\|^{2}$ for $i \neq 0 \bmod k$,

2. $D(l) \leq(\alpha / \delta)^{k^{2}} D(l+1)$ for $l=1, \ldots, m-1$,

3. $\quad \delta^{k^{2}}\left\|\widehat{b}_{k l}\right\|^{2} \leq \alpha\left\|\widehat{b}_{k l+1}\right\|^{2}$ for $l=1, \ldots, m-1$.

Theorem 4. [KS01] Let $b_{1}, \ldots, b_{n}$ be a basis that is $k$-segment LLL-reduced with $\delta$. Then we have for $i=1, \ldots, n$ :

$$
\delta^{2 k^{2}+n-1}\left\|b_{i}\right\|^{2} \leq \alpha^{n-1} \lambda_{i}^{2} \text { and } \delta^{k^{2}+i-1}\left\|b_{1}\right\|^{2} \leq \alpha^{i-1}\left\|\widehat{b}_{i}\right\|^{2},
$$

where $\lambda_{1} \leq \cdots \leq \lambda_{n}$ are the successive minima of the lattice.

Algorithm for segment LLL-reduction. The algorithm segment LLL transforms a given basis into a $k$-segment reduced basis using exact integer arithmetic. It iterates local LLL-reduction of two segments $\left[B_{l-1}, B_{l}\right]=\left[b_{k l-k+1}, \ldots, b_{k l+k}\right]$ via

the procedure loc-L $\mathbf{L L}(l)$. Given the orthogonalization of a $k$-segment reduced basis $b_{1}, \ldots, b_{k l-k}$ the procedure loc-LLL $(l)$ computes the orthogonalization and size-reduction of the segments $B_{l-1}, B_{l}$. In particular it provides the submatrix $R_{l} \in \mathbf{R}^{2 k \times 2 k}$ of $R \in \mathbf{R}^{d \times n}$ corresponding to the segments $B_{l-1}, B_{l}$. Thereafter it performs a local LLL-reduction of $R_{l}$ and stores the LLL-transformation in the matrix $H \in \mathbf{Z}^{2 k \times 2 k}$. Finally, it transforms $\left[B_{l-1}, B_{l}\right]$ into the locally reduced segments $\left[B_{l-1}, B_{l}\right] H$ and size-reduces $\left[B_{l-1}, B_{l}\right]$ globally.

\section{Segment LLL}

INPUT $\quad b_{1}, \ldots, b_{n} \in \mathbf{Z}^{d}, k, m, n=k m, \delta$

OUTPUT $b_{1}, \ldots, b_{n} \quad k$-segment LLL-reduced basis

1. $l:=1$

2. while $l \leq m-1$ do

loc-LLL $(l)$

if $l \neq 1$ and

$\left(D(l-1)>(\alpha / \delta)^{k^{2}} D(l)\right.$ or $\left.\delta^{k^{2}}\left\|\widehat{b}_{k(l-1)}\right\|^{2}>\alpha\left\|\widehat{b}_{k(l-1)+1}\right\|^{2}\right)$

then $l:=l-1$ else $l:=l+1$.

end

Theorem 5. [KS01] For $k=\Theta(m)=\Theta(\sqrt{n})$ segment LLL performs $O\left(n d \log _{1 / \delta} M\right)$ arithmetic steps using integers of bit length $O\left(\log _{2} M\right)$. 


\section{$7 \quad$ Scaled Segment LLL-Reduction.}

We combine the methods of Sections 4 and 6 to a stable algorithm for segment LLL-reduction. Size-reduction is done against an associated scaled basis. Segment LLL-reduction is guided by the orthogonal vectors of the scaled basis.

Definition 5. We call a basis $b_{1}, \ldots, b_{n} \in \mathbf{Z}^{d} k$-segment sLLL-reduced, if it is $k$-segment LLL-reduced except that $b_{1}, \ldots, b_{n}$ is size-reduced in a weaker sense it is size-reduced against an associated basis $b_{1}^{s}, \ldots, b_{n}^{s}$ with the properties 1.2 .:

1. The first orthogonal vectors $\widehat{b}_{k l-k+1}^{s}$ of segments are nearly equally long:

$$
\left\|b_{1}^{s}\right\| \leq 2^{e_{k l}}\left\|\widehat{b}_{k l-k+1}\right\|=\left\|\widehat{b}_{k l-k+1}^{s}\right\|<2\left\|b_{1}^{s}\right\| \quad \text { for } l=1, \ldots, m .
$$

2. There is a uniform scaling factor $2^{e_{k l}}$ for segment $B_{l}$ so that the coefficients $r_{k l-k+i, k l-k+j}$, for $1 \leq i, j \leq k$ of the $R$-matrix corresponding to $b_{1}, \ldots, b_{n}$ and those corresponding to $b_{1}^{s}, \ldots, b_{n}^{s}$ are proportional with proportionality factor $2^{e_{k l}}$.

The inequalities for $k$-segment LLL-reduced bases in Theorem 4 also hold for $k$-segment sLLL-reduced bases. The weaker size-reduction is not important. In practice $k$-segment sLLL-reduced bases are nearly as good as LLL-reduced bases.

We sketch a procedure loc-sLLL $(l)$ - for local scaled LLL-reduction which replaces loc-LLL $(l)$ within segment $\mathbf{L L L}$. The algorithm segment sLLL iterates local scaled LLL-reduction of two consecutive segments $\left[B_{l-1}, B_{l}\right]=$ $\left[b_{k l-k+1}, \ldots, b_{k l+k}\right]$.

The procedure loc-sLLL $(l)$. Its inputs are a lattice basis, uniform scaling factors $2^{\bar{e}_{k \ell}}$ satisfying $b_{k \ell-j}^{s}=2^{\bar{e}_{k \ell}} b_{k \ell-j}$ for $j=0, \ldots, k-1, \ell=1, \ldots, l-1$. The Householder vectors $v_{1}, \ldots, v_{k(l-1)}$ of $b_{1}, \ldots, b_{k(l-1)}$ and the matrix $R \in \mathbf{R}^{d \times k(l-1)}$ for $\left[b_{1}^{s}, \ldots, b_{k(l-1)}^{s}\right]$ are also given. Local LLL-reduction of $B_{l-1}, B_{l}$ is done in $f p a$ via the local matrix $R_{l} \in \mathbf{R}^{2 k \times 2 k}$.

The procedure loc-sLLL $(l)$

- computes a uniform scaling factor $2^{\bar{e}_{k l}}$ for the two segments $\left[B_{l-1}, B_{l}\right]$

- computes the local matrix $R_{l}$ of $2^{\bar{e}_{k l}}\left[B_{l-1}, B_{l}\right]$ via HRS'

- performs a local LLL-reduction on $\left[B_{l-1}, B_{l}\right]$ using $R_{l}$

- stores the transformation in the matrix $H \in \mathbf{Z}^{2 k \times 2 k}$.

- Upon termination it transforms $\left[B_{l-1}, B_{l}\right]$ into $\left[B_{l-1}, B_{l}\right] H$ in exact arithmetic.

Restarting loc-sLLL $(l)$. Whenever $\|H\|_{\infty}$ surpasses the threshold $2^{15}$ the procedure loc-s $\mathbf{L} \mathbf{L} \mathbf{L}(l)$ is restarted with the transformed segments $\left[B_{l-1}, B_{l}\right] H$. This is necessary as the norm $\|H\|_{\infty}$ directly translates into additional fpa-errors. As the number of restarts is crucial for the running time we carefully prepare $R_{l}$ as to prevent an early restart. We show below how to predict the size of the matrix $H \in \mathbf{Z}^{2 k \times 2 k}$ occuring in the subsequent local LLL-reduction of $B_{l-1}, B_{l}$. We slash $R_{l}$ so that $\|H\|_{\infty} \leq 2^{15}$ holds on the average. The threshold $2^{15}$ is for wired 53-bit $f p a$, for 106-bit $f p a$ we increase the threshold accordingly. 
Computing the matrix $R_{l}$ and $2^{\bar{e}_{k l}}$. First compute via HRS the uniform scaling factor $2^{\bar{e}_{k l}}$ and the first vector $\mathbf{r}_{k l-k+1}$ of $B_{l-1}$ so that $\left\|b_{1}^{s}\right\| \leq 2^{e_{k l}}\left\|\widehat{b}_{k l-k+1}\right\|=$ $\left\|\widehat{b}_{k l-k+1}^{s}\right\|<2\left\|b_{1}^{s}\right\|$. With the same scaling factor $2^{\bar{e}_{k l}}$ compute $\mathbf{r}_{h}$ via HRS' for $h=k l-k+2, \ldots, k l+k$. For local LLL-reduction we have to size-reduce $b_{h}$ against $b_{k l-k+1}, \ldots b_{h-1}$. We generalize Step 3. of HRS' accordingly: perform the same size-reduction steps $b_{h}^{s}:=b_{h}^{s}-\left\lceil\bar{\mu}_{h, j}^{s}\right\rfloor b_{j}^{s}, \quad b_{h}:=b_{h}-\left\lceil\bar{\mu}_{h, j}^{s}\right\rfloor b_{j}$ on $b_{h}^{s}$ against $b_{j}^{s}$ and on $b_{h}$ against $b_{j}$ for $j=k l-k+1, \ldots h-1$. This implies that the local matrix $R_{l}$ of segments $B_{l-1}, B_{l}$ corresponding to $b_{1}, \ldots, b_{n}$ and the $R_{l}$ corresponding to $b_{1}^{s}, \ldots, b_{n}^{s}$ are proportional with the factor $2^{\bar{e}_{k l}}$.

The heuristic for slashing $R_{l}$. We let $r_{i, j}^{l}=r_{k l-k+i, k l-k+j}$ denote the coefficients of the local matrix $R_{l}$. Large values $\left|r_{1,1}^{l} / r_{j, j}^{l}\right|$ have a double negative effect on the stability of loc-sLLL $(l)$. By Lemma 1 the orthogonalization of $b_{j}$ gets inaccurate. Moreover, $\|H\|_{\infty}$ will be large due to Lemma 2 [KS01]. A detailed argument shows that $\|H\|_{\infty}$ is expected to be less than $2^{15}$ if $\max _{j}\left|r_{1,1}^{l} / r_{j, j}^{l}\right| \leq 2^{15}$. This suggests to slash $R_{l}$ so that $\max _{j}\left|r_{1,1}^{l} / r_{j, j}^{l}\right| \leq 2^{15}$.

Slashing the matrix $R_{l}$. Slash all values $\left|r_{j, j}^{l}\right|$ - satisfying $\left|r_{1,1}^{l} / r_{j, j}^{l}\right|<2^{-15}$ to $r_{j, j}^{l}:=\left|r_{1,1}^{l}\right| 2^{-15}$. Moreover, set $r_{h, i}^{l}:=0$ for $h=j, \ldots, 2 k$ and $h<i \leq 2 k$.

$$
R_{l}:=\left[\begin{array}{cccccc}
r_{1,1}^{l} & \cdots & r_{1, j-1}^{l} & r_{1, j}^{l} & \cdots & r_{1, k}^{l} \\
& \ddots & \vdots & \vdots & & \vdots \\
0 & & r_{j-1, j-1}^{l} & r_{j-1, j}^{l} & \cdots & r_{j-1, k}^{l} \\
0 & \cdots & 0 & \left|r_{1,1}^{l}\right| 2^{-15} & & 0 \\
\vdots & & \vdots & & \ddots & \\
0 & \cdots & 0 & 0 & & \left|r_{1,1}^{l}\right| 2^{-15}
\end{array}\right]
$$

We locally LLL-reduce the slashed matrix $R_{l}$, and afterwards we transform $\left[B_{l-1}, B_{l}\right]$ into $\left[B_{l-1}, B_{l}\right] H$. If either $\|H\|_{\infty}>2^{15}$ or if the slashing did effectively change $R_{l}$ we restart the local reduction with the transformed segments $\left[B_{l-1}, B_{l}\right] H$. Note that a restart of $\operatorname{loc}-\mathbf{s} \mathbf{L} \mathbf{L} \mathbf{L}(l)$ also adjusts the uniform scaling factor $2^{\bar{e}_{k l}}$. This method of correction works very well for segment size $k \leq 100$.

\section{Segment sLLL}

INPUT $\quad b_{1}, \ldots, b_{n} \in \mathbf{Z}^{d}, k, m, n=k m, \delta$

OUTPUT $b_{1}, \ldots, b_{n} \quad k$-segment and scaled reduced basis

1. $l:=1$

2. while $l \leq m-1$ do

loc-sLLL $(l)$

if $l \neq 1$ and

$\left(D(l-1)>(\alpha / \delta)^{k^{2}} D(l)\right.$ or $\left.\delta^{k^{2}}\left\|\widehat{b}_{k l}\right\|^{2}>\alpha\left\|\widehat{b}_{k l+1}\right\|^{2}\right)$

then $l:=l-1$ else $l:=l+1$. end 


\section{Performance of Segment sLLL.}

Consider a sample of [GGH]-bases of dimension $n=\nu \cdot 100$ for $\nu=1, \ldots, 8$. The vectors of the input basis consist of integers with $n / 2$ bits. The algorithm uses 106 bit $f p a$ and $\delta=0.99$ respectively $\delta=0.999$. All tests have been performed on $350 \mathrm{MHz}$ PC's, the code of segment sLLL uses the [NTL] computer algebra library. We present the segment size and the average bit length of the reduced basis vectors in Figure 2.

\begin{tabular}{|c|c|c|c|c|}
\hline $\begin{array}{c}\text { Lattice } \\
\text { dimension }\end{array}$ & \multicolumn{2}{|c|}{$\delta=0.99$} & \multicolumn{2}{c|}{$\delta=0.999$} \\
segment size & $l^{\text {av }}$ & segment size & $l^{\text {av }}$ \\
\hline \hline 100 & 25 & 7.9 & 25 & 5.2 \\
\hline 200 & 40 & 13.8 & 40 & 9.4 \\
\hline 300 & 45 & 23.0 & 45 & 14.7 \\
\hline 400 & 50 & 30.5 & 50 & 17.7 \\
\hline 500 & 50 & 34.3 & 56 & 22.6 \\
\hline 600 & 60 & 38.8 & 60 & 27.9 \\
\hline 700 & 60 & 40.9 & 65 & 34.3 \\
\hline 800 & 70 & 46.6 & 70 & 37.0 \\
\hline
\end{tabular}

Fig. 2. segment sLLL reduced bases. $l^{a v}=$ average bit length of the output integers.

The running time increases considerably as $\delta$ approaches 1 . The size of the reduced basis decreases accordingly. Figure 3 shows the time of segment sLLL for $[\mathrm{GGH}]$-bases.

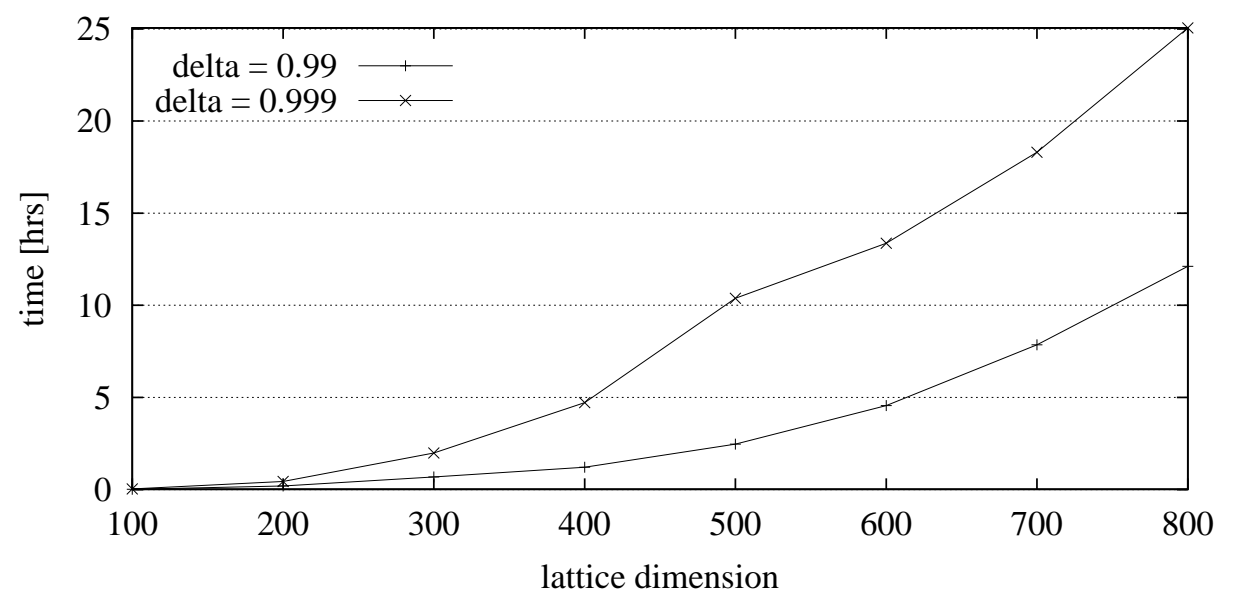

Fig. 3. Running time of Segment sLLL using $\delta=0.99$ resp. $\delta=0.999$.

Acknowledgement. We thank Bartol Filipović for his help in producing and measuring the code of the new reduction algorithm. 


\section{References}

[GGH] O. Goldreich, S. Goldwasser, and S. Halevi, Public-key cryptosystems from lattice reduction problems. Proc. Crypto'97, LNCS 1294, Springer-Verlag, pp. 112-131, 1997.

[KS01] H. Koy and C.P. Schnorr, Segment LLL-Reduction of Lattice Bases. Proceedings CaLC 2001.

[LLL82] A. K. Lenstra, H. W. Lenstra, and L. Lovász, Factoring polynomials with rational coefficients, Math. Ann. 261, pp. 515-534, 1982.

[LH95] C.L. Lawson and R.J. Hanson, Solving Least Square Problems, SIAM, Philadelphia, 1995.

[NTL] NTL homepage: http://www.shoup.net/ntl/, 2000.

[RS96] C. Rössner and C.P. Schnorr, An optimal stable continued fraction algorithm for arbitrary dimension. 5.-th IPCO, LNCS 1084, pp. 31-43, Springer-Verlag, 1996.

[S87] C.P. Schnorr, A hierarchy of polynomial time lattice basis reduction algorithms, Theoretical Computer Science 53, pp. 201-224, 1987.

[S88] C.P. Schnorr, A more efficient algorithm for lattice basis reduction, J. Algorithms 9, pp. 47-62, 1988.

[SE91] C.P. Schnorr and M. Euchner, Lattice Basis Reduction: Improved Practical Algorithms and Solving Subset Sum Problems, Proc. Fundamentals of Computation Theory '91, L. Budach, ed., LNCS 529, Springer-Verlag, pp. 68-85, 1991. (Complete paper in Mathematical Programming Studies 66A, No 2 , pp. 181-199, 1994.)

[Sc84] A. Schönhage, Factorization of univariate integer polynomials by diophantine approximation and improved lattice basis reduction algorithm, Proc. 11-th Coll. Automata, Languages and Programming, Antwerpen 1984, LNCS 172, Springer-Verlag, pp. 436-447, 1984. 